\title{
Thermal Behaviour of Synovene and Oleamide in Oil Adsorbed on Steel
}

\author{
Michael T. L. Casford ${ }^{1}\left[\right.$ D $\cdot$ Debashis Puhan ${ }^{1} \cdot$ Paul B. Davies $^{1} \cdot$ Gareth L. Bracchi ${ }^{2} \cdot$ Tony D. Smith $^{2}$
}

Received: 21 October 2019 / Accepted: 14 March 2020 / Published online: 28 March 2020

(c) The Author(s) 2020

\begin{abstract}
Oleamide and Synovene lubricant additives when mixed together show a clear co-operative effect leading to friction and wear reduction. Sum Frequency Generation vibrational spectroscopy has been used to record in situ spectra of these additives with the aim of understanding the behaviour of these molecules when adsorbed on steel immersed in a model base oil at pre-selected temperatures. The spectra of the individual components and of mixtures have been recorded up to $130{ }^{\circ} \mathrm{C}$. Individual spectra from both molecules have been distinguished using per-deuterated oleamide. The temperature at which maximum ordered adsorption of pure Synovene molecules occurs drops from $\sim 130$ to $\sim 70{ }^{\circ} \mathrm{C}$ in the mixture with oleamide. Our results show that co-adsorption occurs, which causes a change in net polar orientation of the oleamide component suggesting the hydrocarbon chains of the oleamide molecules reverse their polar orientation when Synovene is present. The net effect of co-adsorption and change in orientation as well as conformation of the two molecules could explain the reduction of friction and wear observed at the metal-metal interface.
\end{abstract}

\section{Introduction}

Small amounts of friction modifiers and anti-wear additives added to lubricating oil play a vital role in improving friction and wear performance in tribological contacts, resulting in reduced fuel consumption and lower carbon emission both of which are desirable environmental goals [1, 2]. The application of analytical surface science techniques in determining the structure of the protective layers formed by such additives in model lubricants (additive and additive combinations in base oil) under thermal and tribological conditions is important for gaining a better understanding of lubricant performance [3, 4]. Two additives with proven anti-wear properties used in commercial lubricating oil are Synovene and oleamide, which have shown significant reductions in

Electronic supplementary material The online version of this article (https://doi.org/10.1007/s11249-020-01293-x) contains supplementary material, which is available to authorized users.

Michael T. L. Casford

mtlc2@cam.ac.uk

1 Department of Chemistry, University of Cambridge, Lensfield Road, Cambridge CB2 1EW, UK

2 Technology Centre, Castrol Ltd, Pangbourne, Reading RG8 7QR, UK wear in engine testing. Our aim is to understand the friction and wear reduction mechanism at a molecular level using the non-linear optical technique of Sum Frequency Generation (SFG) spectroscopy [5].

Synovene is a natural product consisting of a 1:3 di-ester formed from glycerol, citric acid and palm oil (a mixture of fatty acids comprising mainly palmitic (C16) and oleic (C18) acids) whilst oleamide has a single aliphatic chain with a cis double bond at its mid-point and an amide terminal group. In the present work, changes in the adsorption of Synovene and oleamide onto steel on their own and from mixtures in base oil as a function of increasing temperature are investigated by SFG to monitor the structural behaviour of their aliphatic chains.

In SFG, two pulsed laser beams, one at a fixed visible frequency and the other tuneable in the infrared region, are temporally and spatially overlapped at an interface such as at a solid/liquid interface. The sum of the two frequencies is monitored as the infrared frequency is swept. When the infrared frequency coincides with a vibrational mode of a molecule present at the interface, there is a change in the intensity at the sum of the two input frequencies. Monitoring the sum frequency provides a vibrational spectrum of the interface molecules upshifted into the visible region of the electromagnetic spectrum where detectors are more sensitive. SFG is interface specific and non-invasive and 
has proven to be particularly useful for detecting the $\mathrm{C}-\mathrm{H}$ stretching modes of hydrocarbon films at interfaces. Generally only molecules adsorbed at the interface produce an SFG spectrum. There is no contribution from bulk phases above the surface, so it is feasible to detect adsorbed molecules in the presence of a liquid over-layer provided the liquid is transparent to the laser and SFG beams, such as the oil film used in the present experiments.

Specific surface selection rules apply for SFG spectra to be generated. First, molecules at the interface must have a net polar orientation. The functional groups of interest here are the terminal methyl groups and the more numerous methylene groups of the aliphatic chains. The intensity of the SFG spectrum is modified by factors such as surface coverage and molecular tilt angles. A second SFG selection rule requires the adsorbed molecules to be both infrared and Raman active. A consequence of this selection rule is that it is possible to differentiate between structures where the aliphatic chains in an adsorbate are conformationally well-ordered, i.e. whether the methylene groups of the chain are all trans, or are disordered with gauche defects in the chain. Only in disordered chains where there is a breakdown in the methylene local symmetry are methylene groups, both infrared and Raman active. Hence a SFG spectrum where the methylene resonances are prevalent indicates disordered hydrocarbon chains.

Recently SFG spectroscopy was used to investigate the adsorption of Synovene on steel and on ZDDP/steel wear tracks from solutions in per-deuterated oil [6]. The detection of SFG spectra in both cases meant that not only was Synovene adsorbed at the oil/metal and at the oil/ZDDP/ metal interfaces but that the aliphatic chains of Synovene had a net polarisation order with respect to the surface. An intense spectrum was observed when the film was initially formed near the melting point of Synovene $\left(\sim 17-27^{\circ} \mathrm{C}\right)$. This was attributed to the formation of an ordered multilayer. This intense initial spectrum decreased in intensity with increasing temperature, most likely due to a decrease in multilayer film thickness and crystallinity, thinning to a monolayer film. The present work is aimed at investigating how the adsorption of Synovene on steel may be modified in the presence of oleamide. At first, the SFG spectra of Synovene and oleamide were recorded individually on steel under oil as the temperature was raised incrementally to $130{ }^{\circ} \mathrm{C}$, followed by recording spectra of their mixtures at oil/steel interface over the same temperature range. Previous work done by us has shown that both Synovene and oleamide reform on steel surfaces within a time frame of less than half of the rotation rate of the cam shaft. This suggests that the static adsorption of both materials is of some relevance to its lubricating action. Further work is ongoing to ascertain the changes in structure under shear.

\section{Experimental}

Synovene, oleamide and per-deuterated oleamide (d-oleamide) solutions were made up in hexadecane (Aldrich) or perdeuterated hexadecane (QMX isotopes, $98 \%$ isotopic purity). The concentration of oleamide used was $0.1 \%$, the maximum solubility of oleamide in hexadecane, and $0.6 \%$ for Synovene by weight. (Higher concentration of Synovene were used to produce SFG spectra of this additive with satisfactory signal to noise ratios.) Diamond polished $(0.1 \mu \mathrm{m}) 1018$ mild steel coupons supplied by European Corrosion Supplies Ltd. (Kidderminster, UK) were used as the substrates and washed in acetone and Decon solution, and UV ozone cleaned for $40 \mathrm{~min}$ to remove hydrocarbon contamination from the atmosphere. For SFG measurements, a thin layer of additive-in-oil solution was trapped on top of the substrate (coupon) by a hemi-cylindrical $\mathrm{CaF}_{2}$ prism used to transmit the input and sum frequency beams. A cartridge heater in close contact with the substrate was used to change the temperature of the coupon, the oil solution and the prism. The temperature was measured with a calibrated k type thermocouple integral to the experimental cell.

SFG spectra in the $\mathrm{C}-\mathrm{H}$ or $\mathrm{C}-\mathrm{D}$ stretching region were recorded with a picosecond spectrometer (EKSPLA), described in detail elsewhere [7]. Briefly, co-propagating visible $(532 \mathrm{~nm})$ and infrared laser beams $(\sim 29 \mathrm{ps}, 50 \mathrm{~Hz})$ were incident on the bare steel. Both $\mathrm{S}$ and $\mathrm{P}$ polarisations with respect to the surface normal could be selected for visible, infrared and sum frequency beams. The two polarisation combinations used here were SSP and PPP (sum frequency, visible, infrared). As a consequence of the reflectivity of metals to infrared and visible light the intensity of the SFG spectra in the PPP polarisation are generally greater than those in the SSP polarisation. Furthermore, the SSP polarisation only generates spectra from molecules with a net polarisation along the surface normal, whereas PPP spectra can arise from molecules both perpendicular and parallel to the surface.

The SFG signal was recorded every $2 \mathrm{~cm}^{-1}$ and all the spectra shown are three-point averages of the experimental data. At least four repeats of each sequence of temperature spectral scans were recorded for both polarisation combinations. Although unlikely to identify any possible contribution from the prism/oil interface, the SFG spectrum of a Synovene solution was recorded with the infrared laser frequency set to $2940 \mathrm{~cm}^{-1}$, the position of the most intense Synovene resonance, with the metal substrate well displaced by $\sim 5 \mathrm{~mm}$ from the prism. No significant SFG signal was detected eliminating any contribution from the prism/oil interface.

To confirm that, a co-operative interaction was present in the model system used here; the coefficient of 
friction and wear data were recorded on a high frequency reciprocating rig (PCS Instruments, UK). Experimental parameters were set to $400 \mathrm{~g}$ loading equivalent to $\sim 1 \mathrm{GPa}$ Hertzian peak pressure, steel-on-steel contact (AISI52100, $800 \mathrm{HV}, 210 \mathrm{GPa}$ ) at $50^{\circ} \mathrm{C}$. Stroke length was $1000 \mu \mathrm{m}$ at $50 \mathrm{~Hz}$ with a ball diameter of $6 \mathrm{~mm}$ for pure hexadecane, $0.1 \%$ oleamide, $0.6 \%$ Synovene and a mix of $0.1 \%$ oleamide and $0.6 \%$ Synovene all in hexadecane.

\section{Results}

\subsection{Friction and Wear Measurements}

Both oleamide and Synovene individually show a clear reduction in the measured coefficient of friction $(\mathrm{CoF}) \mathrm{com}$ pared to the hexadecane base oil. The variation in coefficient of friction is shown in Fig. 1. However, in combination there is an additional decrease in the CoF indicating a co-operative effect of the additive mix within the initial 20 min rubbing contact suggesting that the mode of action of the additives in the model system is consistent with that observed in the engine tests of fully formulated oils. The wear volume loss with Synovene and its mixture with oleamide is one order lower than with oleamide alone; however, due to the extremely small wear volume, the difference observed between Synovene alone and the 6 to $1 \mathrm{mix}$ is not statistically significant. (see Supplementary information SI).

\subsection{Oleamide and Synovene Adsorbed Separately on Steel}

Figure 2 shows the SFG spectra of oleamide adsorbed onto a steel coupon surface from a solution in d-oil recorded in the PPP and SSP polarisation combinations as the temperature

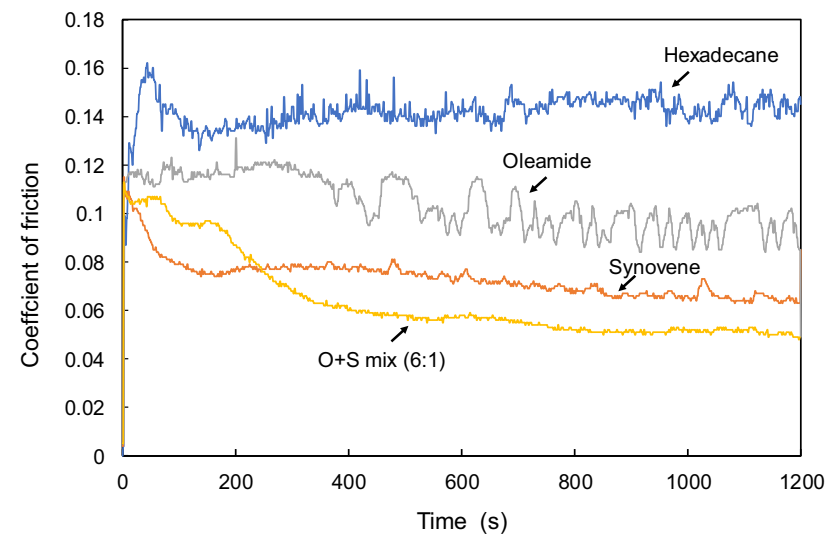

Fig. 1 Variation in the coefficient of friction for hexadecane (base oil), oleamide, Synovene and a 1:6 mix of oleamide and Synovene with time at $50{ }^{\circ} \mathrm{C}$
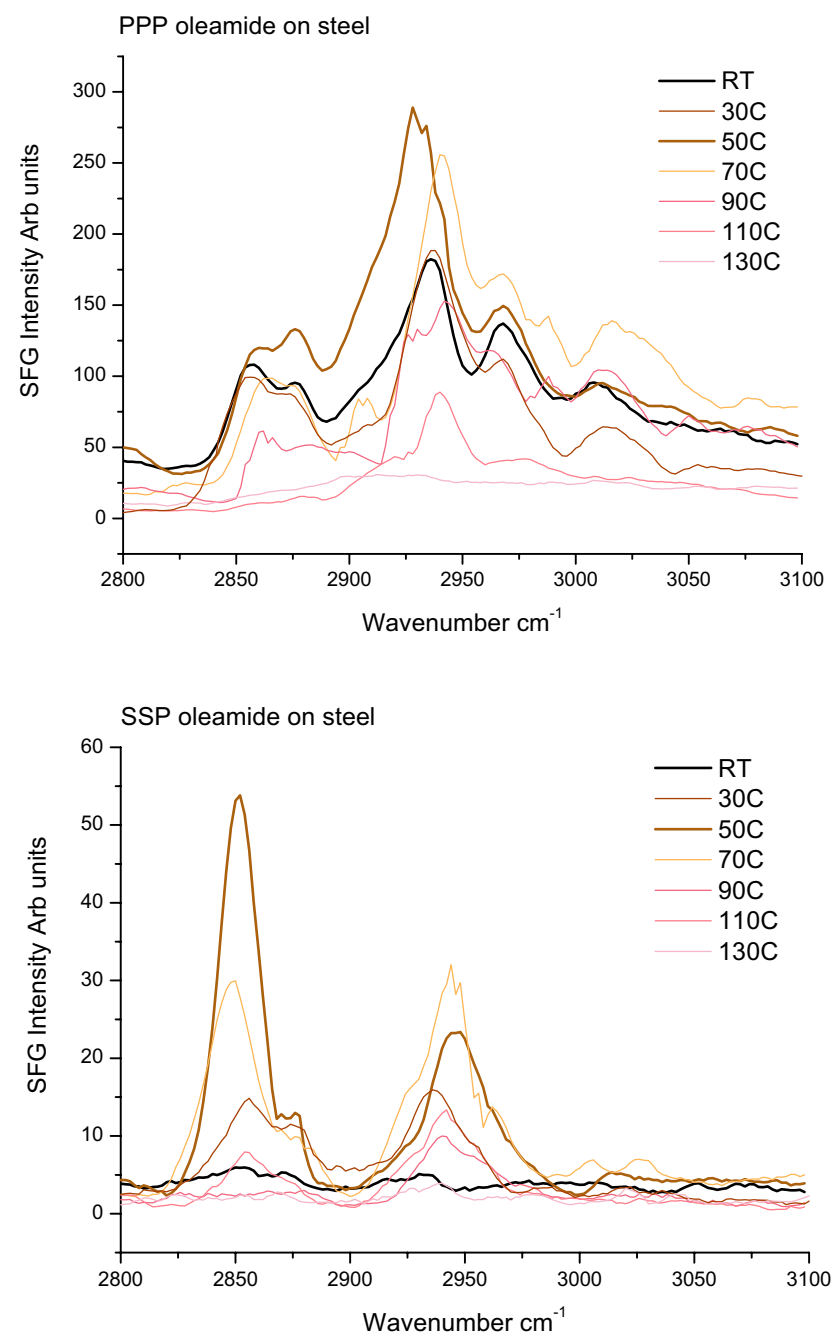

Fig. 2 PPP (upper panel) and SSP (lower panel) spectra in the $\mathrm{C}-\mathrm{H}$ stretching region recorded after a $0.1 \%$ oleamide in d-oil (hexadecane) solution was adsorbed on steel at sequentially increasing temperatures

was increased. SFG spectra in the $\mathrm{C}-\mathrm{H}$ region from the long aliphatic chains of surfactants adsorbed at solid/liquid and air/liquid interfaces have been widely reported in the literature and the resonances in Fig. 2 can be attributed to the aliphatic chain of oleamide. The spectral assignment is shown in Table 1 where the d resonances arise from methylene $\left(\mathrm{CH}_{2}\right)$ groups and $\mathrm{r}$ resonances from methyl $\left(\mathrm{CH}_{3}\right)$ groups and where + superscripts denote symmetric stretching modes and - superscripts asymmetric ones:

The most intense spectra in both polarisation combinations occur around $70{ }^{\circ} \mathrm{C}$. The resonances with the predominant intensity in both polarisations are the $\mathrm{d}^{+}$and $\mathrm{r}_{\mathrm{FR}}{ }^{+}$ although their ratio is not constant at different temperatures. It can be seen from Fig. 2 that there are noticeable variations in the relative intensities of all the spectral features comparing one temperature spectral scan to another. Furthermore, 
Table 1 Assignment of SFG vibrational resonances

\begin{tabular}{lll}
\hline Peaks $\left(\mathrm{cm}^{-1}\right)$ & Assignment & Denoted as \\
\hline 2855,2102 & Sym methylene stretch & $\mathrm{d}^{+}$ \\
2878,2075 & Sym methyl stretch & $\mathrm{r}^{+}$ \\
2900 & Sym methylene stretch Fermi res & $\mathrm{d}_{\mathrm{FR}}{ }^{+}$ \\
2915,2182 & Asym methylene stretch & $\mathrm{d}^{-}$ \\
$2942,2120,2131$ & Sym methyl stretch Fermi res & $\mathrm{r}_{\mathrm{FR}}{ }^{+}$ \\
2966,2222 & Asym methyl stretch & $\mathrm{r}^{-}$ \\
3010,2250 & Olefinic CH/CD stretch (CH/CD & \\
& adjacent to the double bond) & \\
\hline
\end{tabular}

the resonances are not clearly resolved. These observations can be attributed to two factors: the wide variation in orientation angles that can be adopted by the aliphatic chain of oleamide and the many conformations that the chain may adopt at different temperatures. This is in marked contrast with the much narrower resonances found in the SFG spectra of the aliphatic chains of well-packed self-assembled monolayers of molecules like octadecane thiol [8].

In Fig. 2, the difference in room temperature scans and maximum intensity temperature scans is very clear. Additionally, to clarify the temperature dependence, the integrated intensity of the complete SFG spectrum was calculated at each temperature and plotted as bar charts (see Fig. 3). The overall integrated intensity of the SFG spectra is an approximate measure of the degree of molecular (polar and conformational) ordering on the surface. In well-ordered systems the intensity of the resonances from the methyl and methylene modes have a different tilt angle dependency. Consequently, the tilt angle can be determined from an analysis of the relative peak intensities, in particular of the

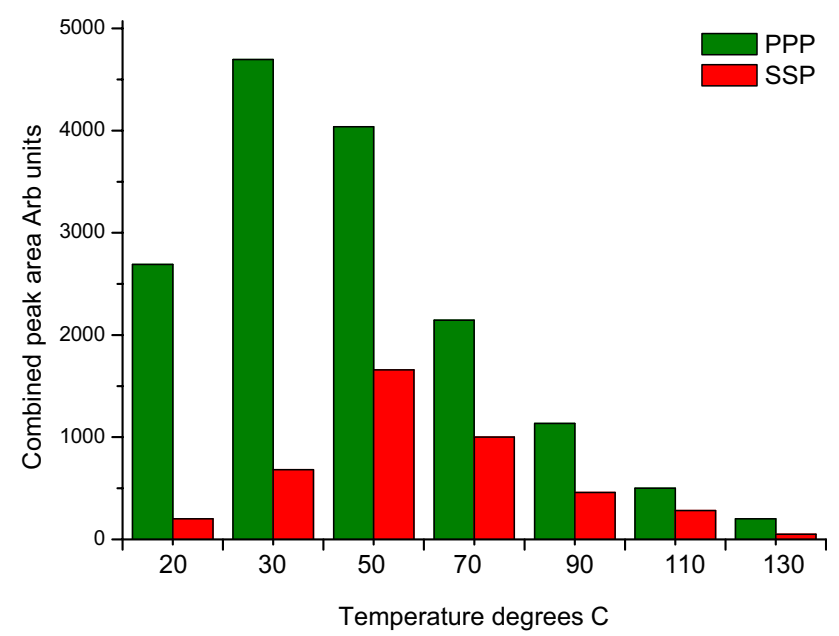

Fig. 3 Bar chart of the integrated intensities of the SFG spectra of oleamide adsorbed on steel as a function of temperature (the room temperature data are displaced to the left for clarity) methyl symmetric and anti-symmetric modes within a given polarisation combination. However, for more disordered systems as is the case here, the spectra are predominantly due to the methylene modes. The overall intensity of the spectra can then be approximately equated to the degree of ordering on the surface. The data in Fig. 3 qualitatively support the conclusion that the temperature has a significant influence on the molecular ordering and the maximum intensity of the spectra. It is possible that the change in the molecular ordering influences the friction experienced at the interface. Figure 4 shows the variation of the average of the friction coefficients recorded during the entire test, as a function of temperature for oleamide, Synovene and their 6:1 mixture. (Steady-state and time-averaged breakdown of this result with error bars is given in Supplementary Information Figure S-4.) The disordering in oleamide molecules may cancel out the effect of temperature on friction on a macroscale, thereby causing the average friction to be similar at all three temperatures tested.

It is clear from an inspection of the oleamide PPP spectra that there is evidence of the olefinic $\mathrm{C}-\mathrm{H}$ stretching mode at $\sim 3010 \mathrm{~cm}^{-1}$ at temperatures below $110{ }^{\circ} \mathrm{C}$, an effect that is also seen in the per-deuterated version of oleamide (shown later in Fig. 10) suggesting that the per-deuteration does not significantly perturb the thermal response of oleamide on its own. However, as the temperature is increased, both the olefinic stretch and the methyl $\mathrm{r}^{+}$stretch decrease in intensity relative to the other stretching modes. This is indicative of an interaction of the double bond with the steel surface that is lost at elevated temperature, implying that the $\mathrm{C}-\mathrm{C}$ double bond becomes disordered between 90 and $110{ }^{\circ} \mathrm{C}$, whilst the

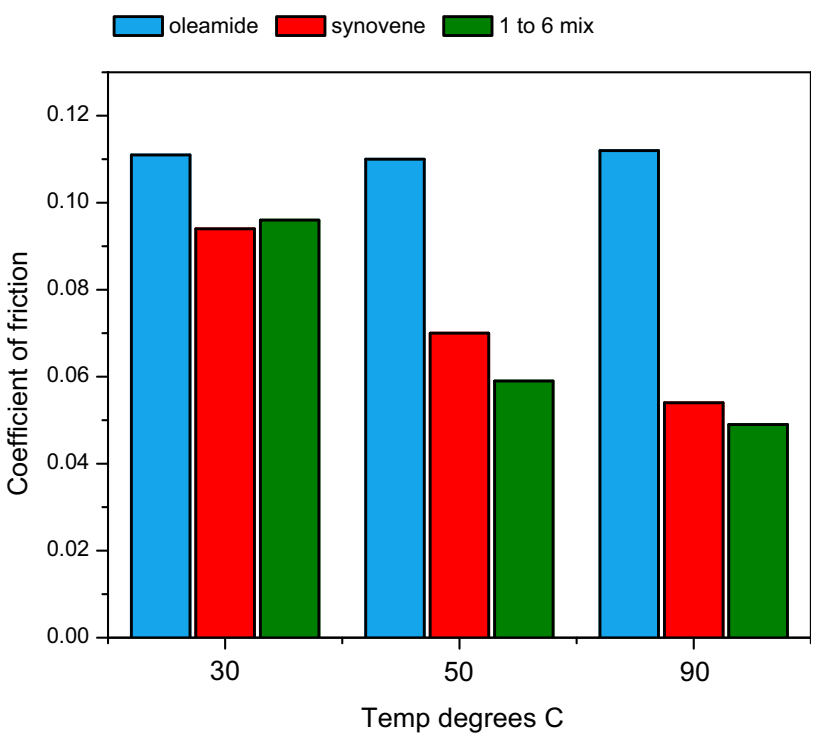

Fig. 4 Variation of time-averaged friction coefficient as a function of temperature 
orientation of the whole of the alkyl chain becomes completely randomised by $130{ }^{\circ} \mathrm{C}$.

Figure 5 shows the corresponding temperature-dependent spectra for the adsorption of Synovene in d-hexadecane on steel. The same spectral assignments apply as for oleamide. The temperature sequence was begun at a slightly higher temperature than the room temperature spectrum because very high intensity spectra are observed at the ambient temperature of the laboratory $\left(16-17^{\circ} \mathrm{C}\right)$ attributed to multilayer formation. This observation is described in more detail in [6]. Not surprisingly, given the different structures of the two molecules, there are differences in the spectra of the two molecules although the $\mathrm{d}^{+}$and $\mathrm{r}^{+}{ }_{\mathrm{FR}}$ resonances remain prominent in both polarisations. We note that a similar spectral intensity has previously been observed by Zhang et al. for an alkane thiol monolayer on mild steel where it was attributed to either a breakdown product or an oxidation effect on the steel surface [9]. Synovene shows a similar increase in conformational disordering of the alkyl chain with temperature.
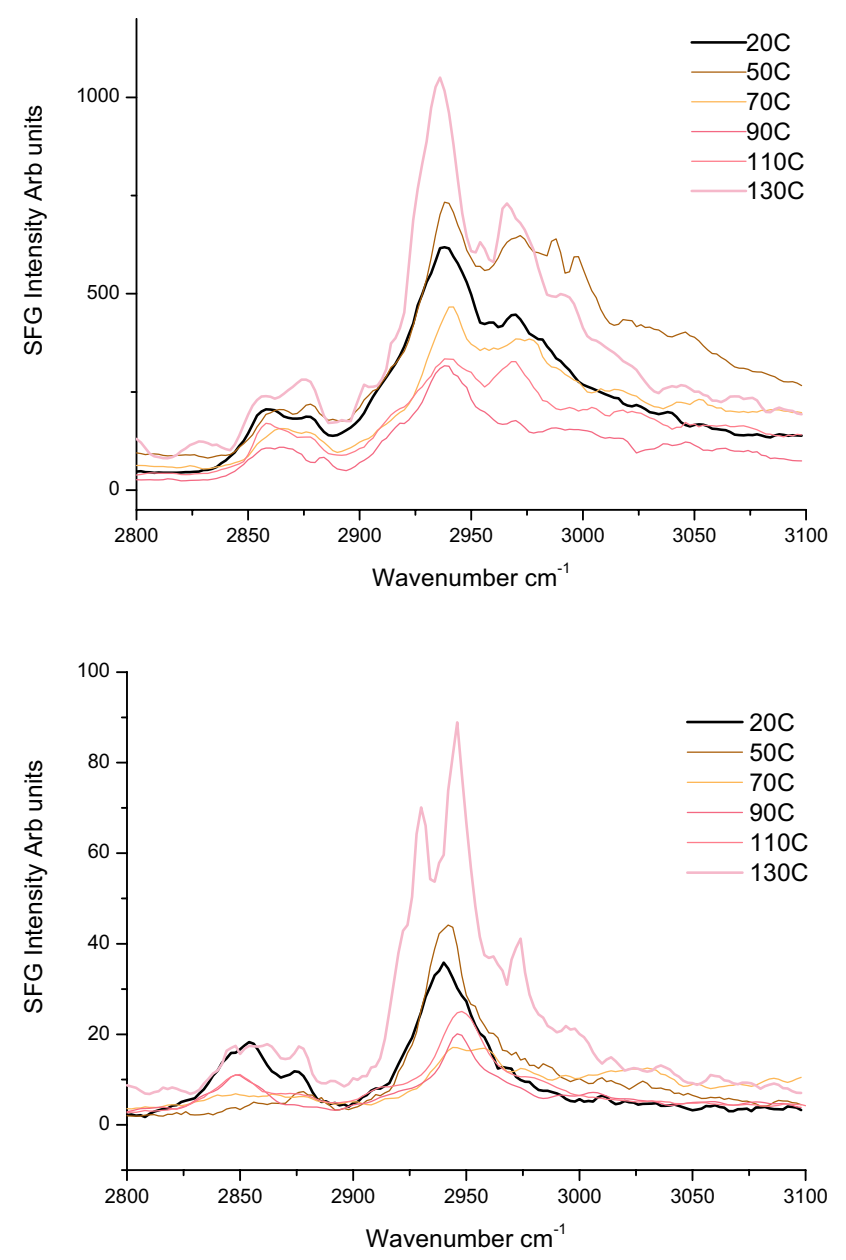

Fig. 5 PPP (upper panel) and SSP (lower panel) spectra in the $\mathrm{C}-\mathrm{H}$ stretching region recorded after a $0.6 \%$ Synovene in d-oil solution was adsorbed on steel at sequentially increasing temperatures
Whilst the overall profiles of the PPP spectra of adsorbed oleamide and Synovene are qualitatively similar, the SSP spectra of the two molecules are markedly different. Furthermore, as can be seen by comparing Figs. 2 and 5, the sequential changes in intensity with temperature are different and the maximum intensity occurs at a markedly higher temperature for Synovene than for oleamide. Hence, oleamide resonances have become very weak at $110^{\circ} \mathrm{C}$ disappearing completely by $130^{\circ} \mathrm{C}$, whereas Synovene resonance intensities at first fall with increasing temperature and then rapidly increase to a maximum at $130{ }^{\circ} \mathrm{C}$. Figure 6 shows the integration of the SFG resonance intensities versus temperature corresponding to the data for oleamide in Fig. 5. These SFG observations mean that not only are Synovene molecules still present on the surface at the highest temperature but that they are adsorbed with a net polar orientation with respect to the surface. This may be a significant factor contributing towards the decrease in the friction as temperature increases (see Fig. 4) when Synovene is present on its own.

\subsection{Adsorption from Oleamide and Synovene Mixtures in Oil}

Next to investigate any changes in the adsorption of Synovene in the presence of oleamide, spectra of mixtures of Synovene and d-oleamide in d-hexadecane in the ratios of $6: 1,3: 1$ and $1: 1$ were recorded at temperatures up to $130{ }^{\circ} \mathrm{C}$. The spectra for the 6:1 ratio are shown in Fig. 7. In the $\mathrm{C}-\mathrm{H}$ region, $\mathrm{SFG}$ spectra in the mixture must arise from the Synovene component and may be compared with the spectra of Synovene on its own in Fig. 5. The corresponding bar diagrams are Figs. 8 and 6, respectively. It

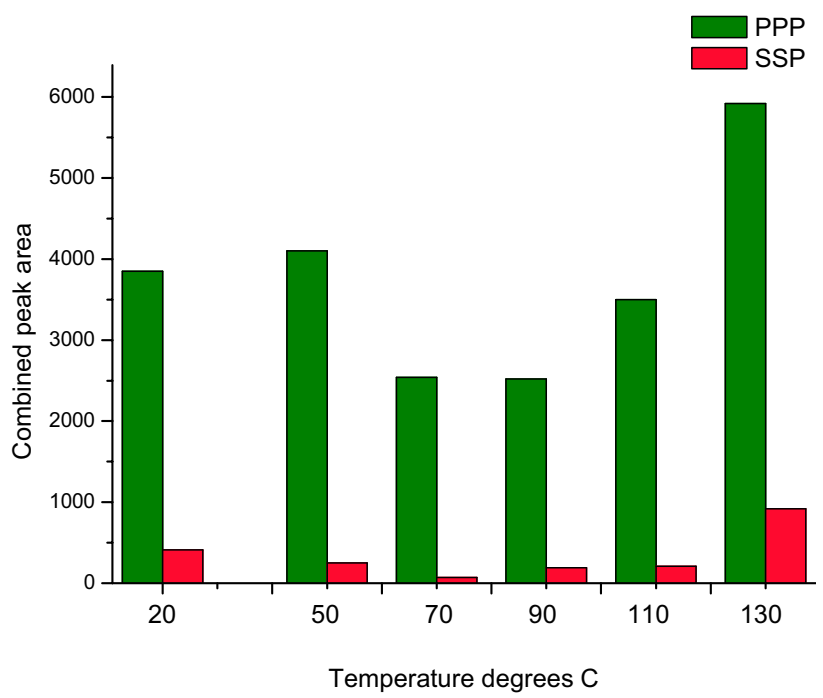

Fig. 6 Total SFG intensity of Synovene adsorbed on steel as a function of temperature (the room temperature data are displaced to the left for clarity) 
1:6 d oleamide:synovene on steel PPP

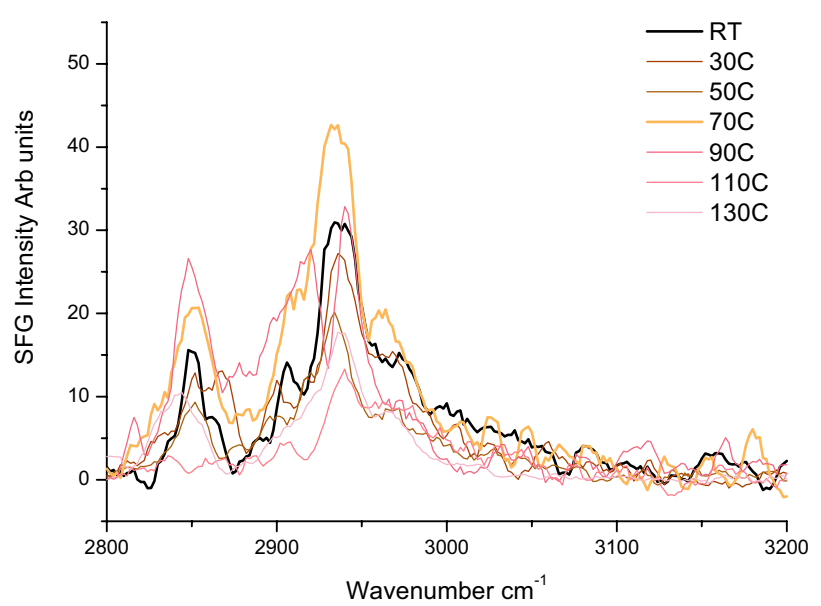

1:6 d oleamide:synovene on steel SSP

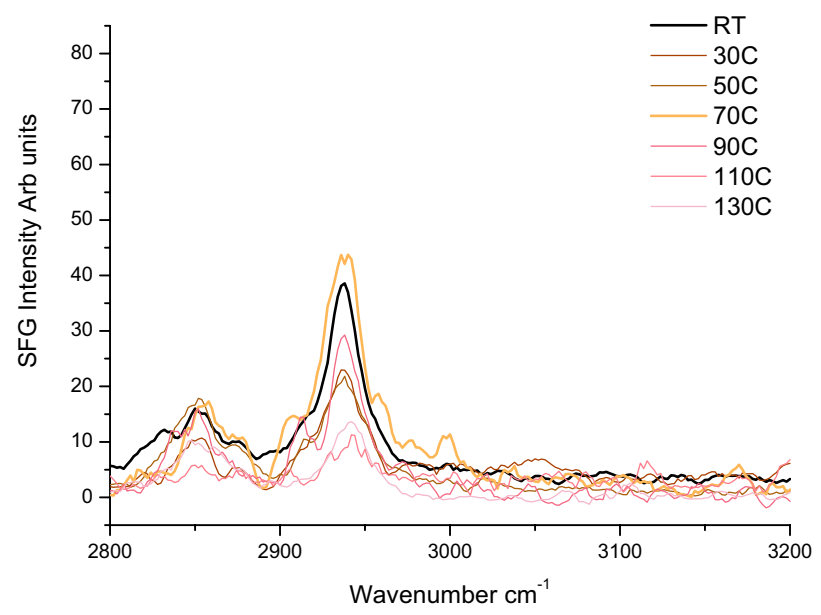

Fig. 7 PPP (upper panel) and SSP (lower panel) spectra of the adsorbed layer formed on steel from a 6:1 Synovene: d-oleamide mixture in d-oil as the temperature is raised from room temperature to $\sim 130{ }^{\circ} \mathrm{C}$

can be seen that there are significant differences between the spectra of Synovene alone and of Synovene in the mixture particularly in the PPP spectra, most notable is the increase in the methylene $2850 \mathrm{~cm}^{-1}$ resonance intensity relative to that of the Fermi resonance at $2940 \mathrm{~cm}^{-1}$. In the light of the findings of Zhang et al., this may suggest that there is a change in the interaction of the adsorbed friction modifiers and the iron oxide surface film. However, considerable additional work would be required to confirm this. Furthermore, the temperature at which the intensity is a maximum drops from $130{ }^{\circ} \mathrm{C}$ (or higher) in pure Synovene adsorption to around $70{ }^{\circ} \mathrm{C}$ in the mixture. This is confirmed by the bar charts, Figs. 6 and 8 . The observed spectra in the more Synovene dilute solutions (not shown) indicate a reduction in ordered Synovene adsorption as the mixing ratio is decreased. Most notably, there is little

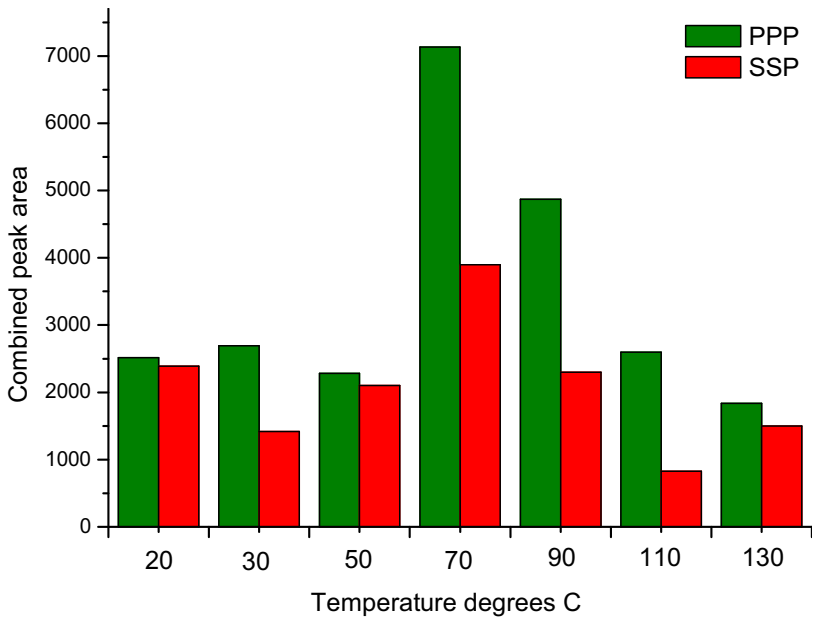

Fig. 8 PPP and SSP bar chart showing total integrated peak area of the spectra from an adsorbed layer formed on steel from a 6:1 Synovene: $\mathrm{d}$-oleamide mixture in $\mathrm{d}$-oil as the temperature is raised from room temperature to $\sim 130{ }^{\circ} \mathrm{C}$

evidence for Synovene adsorption on the steel surface at all in the $1: 1 \mathrm{mix}$ at temperatures above $70{ }^{\circ} \mathrm{C}$.

To investigate the behaviour of the deuterated oleamide component in the mixture, spectra were recorded in the C-D stretching region and are shown in both polarisations in Fig. 9. The most notable feature is the change of phase direction in the resonances in both cases. Since the phase of the d-oleamide signals is now opposite to that of the Synovene, this suggests that their SFG active $\mathrm{C}-\mathrm{H}$ functional groups are pointing in opposite directions on the surface but still orientated along the surface normal. This switching of phase and hence of net oleamide polar orientation when Synovene is present suggests that the structural reorientation of oleamide has been caused by the presence of Synovene.

To further ascertain the effect of Synovene on oleamide, the spectra in the $\mathrm{C}-\mathrm{D}$ region of only d-oleamide in hexadecane were recorded and are shown in Fig. 10. The similar phase of the spectra in Figs. 10 and 2 shows that d-oleamide adsorbed on its own behaves the same as oleamide on its own and thus it is confirmed that Synovene has a detectable effect on the orientation of d-oleamide (and oleamide) when present in a mixture. This change in orientation and relative adsorption of the molecules could lead to lowering of the boundary friction as seen in Figs. 1 and 4, and of the wear (see Supplementary Information S1).

The weaker d-oleamide spectra in Fig. 10 compared with Fig. 9 supports the result from Figs. 8 and 11, namely that the molecules are co-adsorbed. The spectra in Fig. 10 can be assigned from the $\mathrm{C}-\mathrm{D}$ stretching mode positions given in Table 1. The difference in the spectra between 2050 and $2200 \mathrm{~cm}^{-1}$ in the two figures arises from two factors. Firstly, the weaker spectra in the mixture is due to co-adsorption of Synovene which reduces the area occupied by oleamide. 

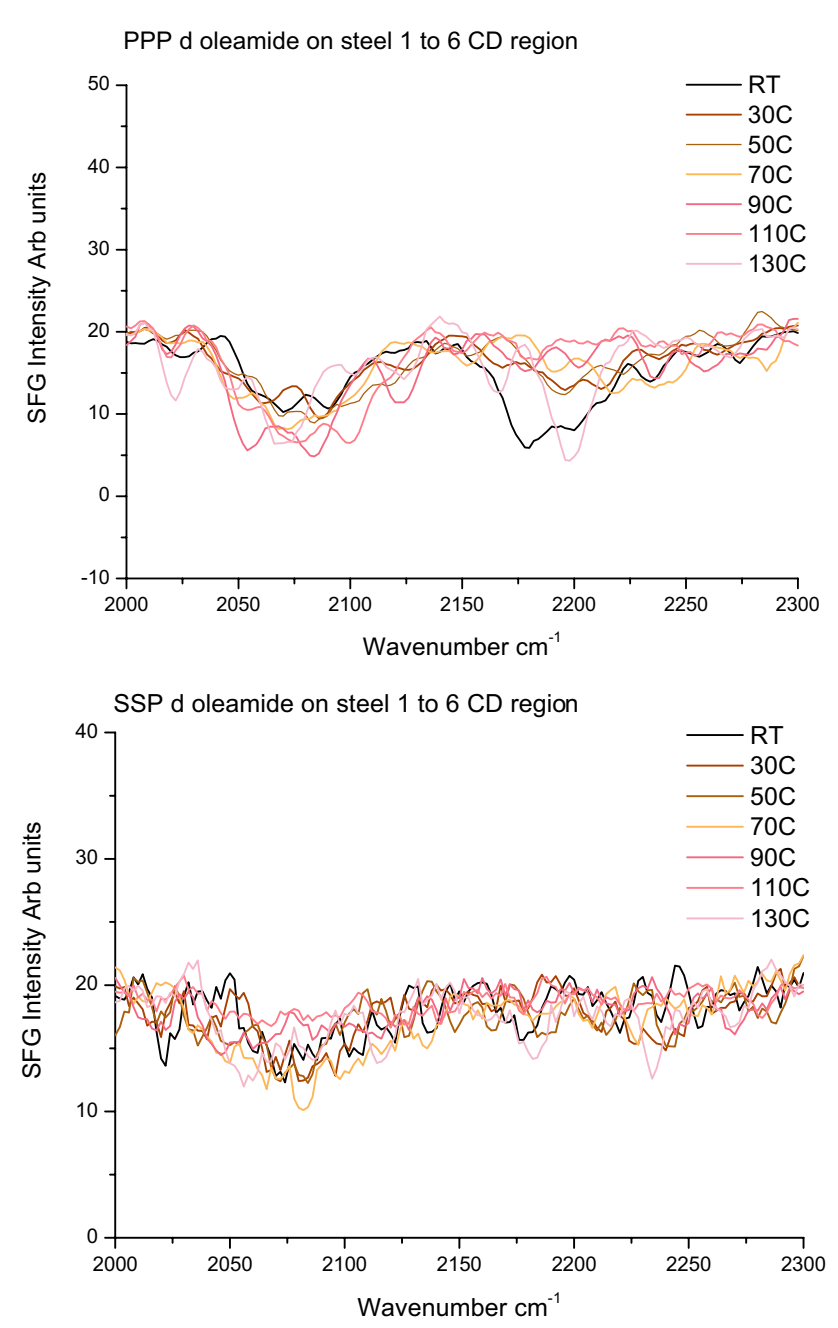

Fig. 9 PPP (upper panel) and SSP (lower panel) spectra in the C-D stretching region of the adsorbed layer formed on steel from a 6:1 Synovene: d-oleamide mixture in d-oil as the temperature is raised from room temperature to $\sim 130{ }^{\circ} \mathrm{C}$

Secondly, the presence of Synovene also changes the orientation and conformation of the oleamide chains giving rise to different relative intensities.

\section{Conclusion}

SFG vibrational spectroscopy has been used to gain insight into the friction and wear reduction mechanisms of two lubricant additives Synovene and oleamide at a molecular level. When these two additives adsorb on steel from a model base oil at temperatures up to $130{ }^{\circ} \mathrm{C}$, at least some parts of their hydrocarbon chains orientate perpendicular to the surface. Based on the spectral intensity of the methyl resonances of the molecules, the maximum ordered adsorption occurs at $\sim 70{ }^{\circ} \mathrm{C}$ for oleamide and at $130{ }^{\circ} \mathrm{C}$ for Synovene. However, the optimum intensity of Synovene spectra in the
PPP $0.1 \%$ d oleamide on steel in $\mathrm{h}$ oil

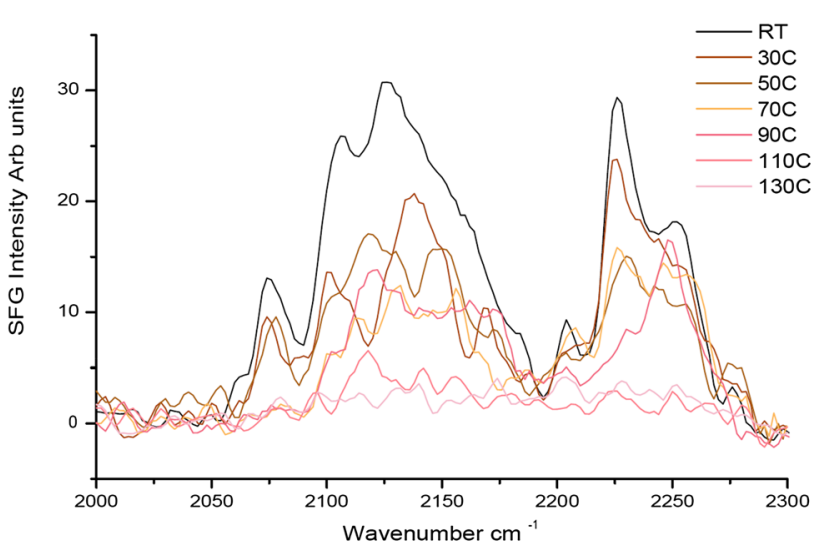

SSP $0.1 \%$ d oleamide on steel in $\mathrm{h}$ oil

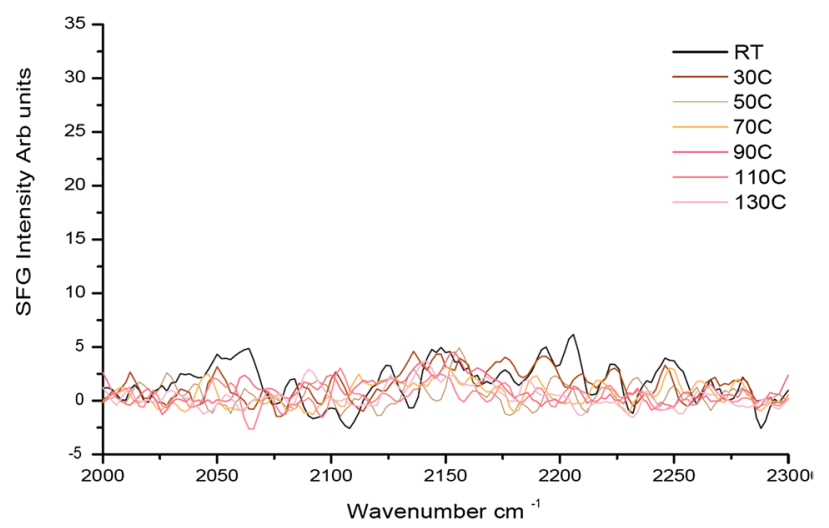

Fig. 10 PPP (upper panel) and SSP (lower panel) spectra in the C-D stretching region recorded after a $0.1 \%$ d-oleamide in h-oil (hexadecane) solution was adsorbed on steel at sequentially increasing temperatures up to $\sim 130{ }^{\circ} \mathrm{C}$

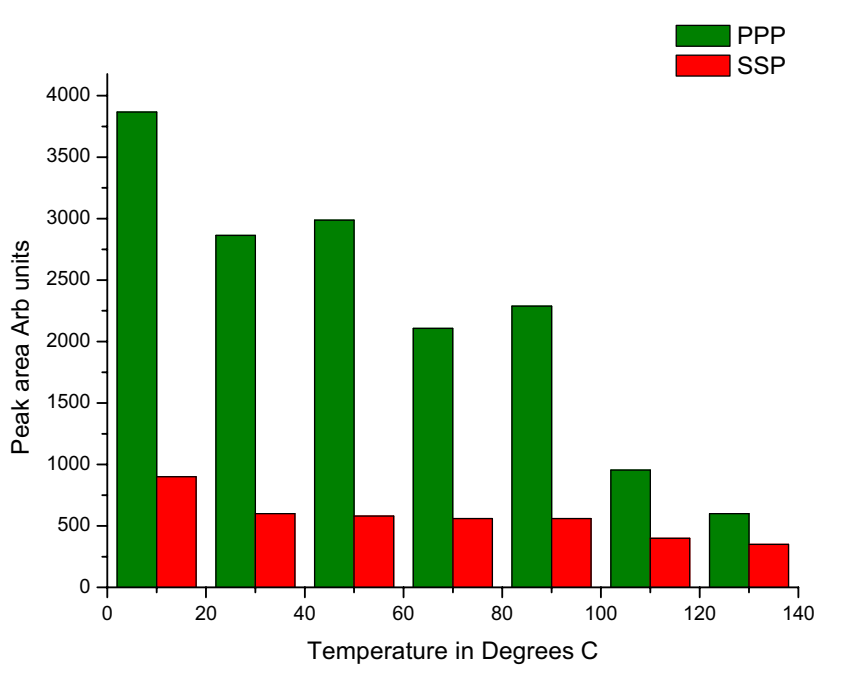

Fig. 11 PPP and SSP bar chart showing total integrated peak area of the spectra (Fig. 9) from an adsorbed layer formed on steel after a $0.1 \%$ d-oleamide in h-oil (hexadecane) solution was adsorbed at sequentially increasing temperatures 
mixture falls to $\sim 70{ }^{\circ} \mathrm{C}$, similar to that of pure oleamide spectra. It is also significant that when mixed spectra from both molecules are detected on the steel surface. The reduction in the intensity of the Fermi resonances in the mixed SFG spectra points to a change in the electronic structure of the iron oxide surface and/or co-operative interaction. If the latter is the case, it suggests the formation of a mixed additive complex at the interface. The co-adsorption results in a change in net polar orientation of the oleamide component implying that the hydrocarbon chains of the oleamide molecules reverse their polar orientation when Synovene is present. We postulate that the reduction in friction and wear at a metal-metal interface when both additives are present as compared to when they are present individually is a direct consequence of co-operative behaviour between both additive molecules.

Acknowledgements We thank Dr Janet Wong of the Department of Engineering, Imperial College London, for providing us with the facilities to make tribological measurements.

Open Access This article is licensed under a Creative Commons Attribution 4.0 International License, which permits use, sharing, adaptation, distribution and reproduction in any medium or format, as long as you give appropriate credit to the original author(s) and the source, provide a link to the Creative Commons licence, and indicate if changes were made. The images or other third party material in this article are included in the article's Creative Commons licence, unless indicated otherwise in a credit line to the material. If material is not included in the article's Creative Commons licence and your intended use is not permitted by statutory regulation or exceeds the permitted use, you will need to obtain permission directly from the copyright holder. To view a copy of this licence, visit http://creativecommons.org/licenses/by/4.0/.
Lubricants. Springer, Dordrecht, pp 320-348 (1997). https://doi. org/10.1007/978-94-017-1021-3_12

2. Minami, I.: Molecular science of lubricant additives. Appl. Sci. 7, 445 (2017). https://doi.org/10.3390/app7050445

3. Koshima, H., Kamano, H., Hisaeda, Y., Liu, H., Ye, S.: Analyses of the adsorption structures of friction modifiers by means of quantitative structure-property relationship method and sum frequency generation spectroscopy. Tribol. Online 5, 165-172 (2010). https://doi.org/10.2474/trol.5.165

4. Watanabe, S., Nakano, M., Miyake, K., Sasaki, S.: Analysis of the interfacial molecular behavior of a lubrication film of n-dodecane containing stearic acid under lubricating conditions by sum frequency generation spectroscopy. Langmuir 32, 13649-13656 (2016). https://doi.org/10.1021/acs.langmuir.6b03954

5. Shen, Y.R.: Basic theory of surface sum-frequency generation. J. Phys. Chem. C 116, 15505-15509 (2012). https://doi.org/10.1021/ jp305539v

6. Casford, M.T.L., Davies, P.B., Smith, T.D., Bracchi, G.L.: The adsorption of synovene on ZDDP wear tracks: a sum frequency generation (SFG) vibrational spectroscopy study. Tribol. Lett. 62, 11 (2016). https://doi.org/10.1007/s11249-016-0662-2

7. Wang, J., Chen, C., Buck, S.M., Chen, Z.: Molecular chemical structure on poly(methyl methacrylate) (PMMA) surface studied by sum frequency generation (SFG) vibrational spectroscopy. J. Phys. Chem. B 105, 12118-12125 (2001). https://doi.org/10.1021/ jp013161d

8. Bain, C.D.: Sum-frequency vibrational spectroscopy of the solid/ liquid interface. J. Chem. Soc. Faraday Trans. 91, 1281-1296 (1995). https://doi.org/10.1039/FT9959101281

9. Zhang, H., Romero, C., Baldelli, S.: Preparation of alkanethiol monolayers on mild steel surfaces studied with sum frequency generation and electrochemistry. J. Phys. Chem. B 109, 1552015530 (2005). https://doi.org/10.1021/jp052807p

Publisher's Note Springer Nature remains neutral with regard to jurisdictional claims in published maps and institutional affiliations.

\section{References}

1. Bovington C.H.: Friction, wear and the role of additives in their control. In: Chemistry and Technology of 